\title{
The relationship between the nurse work environment and delivering culturally sensitive perinatal hospice care
}

\author{
Dr. Sandra J. Mixer, PhD, RN, CTN-A ${ }^{1}$ [Assistant Professor], Dr. Lisa C. Lindley, PhD, RN ${ }^{1}$ \\ [Assistant Professor], Dr. Heather S. Wallace, PhD ${ }^{2}$ [Assistant Professor], Ms. Mary Lou \\ Fornehed, MSN, RN, ACNP-BC ${ }^{1,3}$ [PhD student University of Tennessee Knoxville and \\ Instructor, School of Nursing, Tennessee Tech University, Cookeville, TN], and Dr. \\ Charlotte Wool, PhD, RN ${ }^{4}$ [Assistant Professor] \\ ${ }^{1}$ University of Tennessee - Knoxville, College of Nursing \\ ${ }^{2}$ University of Tennessee - Knoxville, Family \& Consumer Science \\ ${ }^{3}$ Tennessee Tech University, Cookeville, TN, College of Nursing \\ ${ }^{4}$ York College of Pennsylvania, York, PA, Department of Nursing
}

\section{Abstract}

Background-Wide variations exist among perinatal hospices, and barriers to perinatal palliative care exist at the healthcare systems level. Research in the area of culturally sensitive perinatal palliative care has been scarce, a gap which this study addresses.

Objective-To evaluate the relationship between the nurse work environment and the delivery of culturally sensitive perinatal hospice care.

Methods-This retrospective, correlational study used data from the National Home and Hospice Care Survey, which includes a nationally representative sample of hospice care providers. A multivariate logistic regression model was used to estimate the relationship between the delivery of culturally sensitive care and the nurse work environment.

Results-Accreditation, teaching status, and baccalaureate-prepared registered nurse staff impacted the provision of culturally sensitive perinatal care.

Conclusions-The hospice and nursing unit environments, specifically in regards to education and technology, may be important contributors to the delivery of culturally sensitive care.

\section{Keywords}

End of life care; hospice organization; nursing leadership; nursing administration; nursing staff

Integral to the care of medically fragile neonates is the reality that not all will survive. Chromosomal abnormalities or major structural defects occur in approximately $3 \%$ of all live births and are the leading causes of infant mortality (Macdorman,, Kirmeyer, and

Corresponding Author: Sandra J. Mixer, PhD, RN, CTN-A, University of Tennessee, 1200 Volunteer Blvd., Knoxville, TN 37996, smixer@utk.edu, Phone: 865-974-9430, Fax: (865) 974-3569.

Declaration of Conflicts: The authors declare no conflicts of interest with respect to the authorship and/or publication of this article. 
Wilson, 2012). Compounding this challenge are disparities in outcomes, which exist among race, ethnicity, and cultural groups. Recent reports from the Center for Disease Control and Prevention show that differences in birth defects exist based on race and ethnicity. Preterm birth rates are in the range of 16-18\% in black women compared with 5-9\% for white women, reflecting social, cultural, and economic disparities (Centers for Disease Control and Prevention, 2013a). In addition, as immigrant groups spend more time in the U.S., their preterm birth rate increases and contributes to poor neonatal outcomes (Goldenberg, et al., 2008).

Today the majority of diagnoses for several life-limiting disorders occur in the prenatal period (Irving et al., 2011). The technology behind expanded prenatal testing has developed rapidly in the last decade, resulting in increased detection of anomalies and the subsequent need to provide care to families facing an unexpected fetal diagnosis (Hickerton et al., 2012) While technology has contributed to better outcomes for preterm neonates born needing intensive care, preterm-related causes of death together accounted for $35 \%$ of all infant deaths in 2009, more than any other single cause (Centers for Disease Control and Prevention, 2013b).

Perinatal hospice, also called perinatal palliative care, has emerged as an interdisciplinary, comprehensive, coordinated approach to supporting families facing the possibility of perinatal death (Balaguer et al,. 2012; Wool, 2012). Perinatal deaths refer to fetal deaths after 20 weeks gestation and live births with only brief survival (Barfield, 2011). Experts recommend that palliative care begin at the time of prenatal diagnosis and extend into the postpartum period (Sumner, Kavanaugh, and Moro, 2006). In response to this growing need, new perinatal hospices have formed across the United States. Since with $60 \%$ of hospices now offering perinatal hospice services (Lindley, Fornehed and Mixer, 2013).

Anecdotal data among experts in the field suggest that wide variations exist among perinatal hospices, including how services are rendered and who delivers the care. Research has shown that certain characteristics of hospice work environments, such as size, affiliation, ownership, organizational age, and accreditation shape how well a hospice can provide care to infants (Lindley, Fornehed and Mixer, 2013). Although palliative care is an interdisciplinary effort, the Institute of Medicine acknowledges that nurses play a central role, both at the core of the inpatient consulting team and in hospice-based programs (Field and Behrman, 2014). Nurses, often at the forefront of helping parents facing perinatal death, express that barriers to perinatal palliative care exist at the health care systems level. The inability to secure administrative support and access interdisciplinary personnel for team meetings were viewed as significant obstacles (Wool, 2015).

Research in the area of culturally sensitive perinatal palliative care is scarce (Wool, 2013). Cultural aspects of palliative care are addressed in the National Consensus Panel's (NCP) Clinical Practice Guidelines for Quality Palliative Care (National Consensus Panel Clinical Practice Guidelines, 2013). Recommendations include 1) communication in a language parents understand, 2) use of interpreter services, 3) availability of written materials, 4) identification of and referral to community services, and 5) implementation of a culturally relevant plan of care (p. 29). Further, the NCP guidelines state that palliative care programs 
should strive for cultural and linguistic competence to ensure appropriate and relevant services are provided. Cultural care and cultural assessment are multi-dimensional concepts including traditions, faith, family, food, educational, economic, and technical factors, and healthcare practices (McFarland \& Wehbe-Alamah, 2015). Language is an important component of cultural care. The patient's and family's ability to understand written material, as well as what the provider is saying, has great impact on their health and illness (Arthur, et al., 2014; Koh, Gracia, \& Alvarez, 2014; Lion, et al., 2013). In the context of this study, language was the major focus of culturally sensitive care; providing interpreter service, written materials in parents' language, and multilingual staff.

The aim of this study was to evaluate the relationship between the nurse work environment and the delivery of culturally sensitive perinatal hospice care. Investigating differences in the delivery of culturally sensitive care will aid clinicians in examining important practice variations and deficiencies in end-of-life perinatal hospice care. Understanding past practices will help stakeholders plan new approaches to meeting patients' cultural needs and achieving defined benchmarks.

\section{Methods}

\section{Study Design and Sample}

We conducted a retrospective, correlational design study that included hospice and home health care agencies in the United States. Agencies were identified using the most recent data available which was the 2007 National Home and Hospice Care Survey (NHHCS), a complex survey that includes information on a national representative sample of hospice care providers (United States Department of Health and Human Services, 2010). Agencies were included if they were licensed hospice providers, offered perinatal hospice services, and were Medicare/Medicaid certified. Agencies were excluded if they did not employee registered nurses $(\mathrm{RN})$, were a home health care agency only, or had missing data. One thousand thirty six hospices and home health agencies constituted the unweighted sample. After applying inclusion and exclusion criteria, our final weighted sample size was 995 agencies that provided perinatal hospice care. Institutional review board approved as exempt research.

\section{Data Source}

The NHHCS provided detailed national-level agency-reported information on the hospices and home health care providers. The NHHCS is a nationwide survey sponsored and conducted by the Centers for Disease Control and Prevention. Agency directors or their designated staff were interviewed in-person for the survey with questions from an agency and staffing questionnaire. No patients or families were contacted. Data collection procedures and quality information were available in the Centers for Disease Control and Prevention manual (Centers for Disease Control and Prevention, 2011).

\section{Measures}

Outcome variables-The NHHCS survey included a number of questions assessing the delivery of culturally sensitive care in the agency questionnaire. We created three binary 
indicators of delivering culturally sensitive care. Our measure of interpreter service was whether or not an agency provided interpreters. Material translation was defined as the agency provided patient-related materials translated into languages of commonly represented groups in their service area. We operationalized multilingual staff as the agency provided multilingual staff for communication with patients and their families.

\section{Predictor variables}

Hospice Environment-Using data from the agency questionnaire, we created 7 measures of the hospice environment. Hospice affiliation was a category measurement of its freestanding nature (e.g. hospital, home health, or long-term care based). The service area of the perinatal hospice was operationalized as metropolitan, micropolitan, or rurally located. A small agency of $<100$ patients a day or large agency of $>100$ patients per day, determined the facility size variable. Organizational age was determined by the length in years of licensure as a hospice. Ownership was defined by whether a hospice reports its profit status as for profit or non-profit/government. If the hospice was used as a teaching environment to train students, this determined the teaching status. Accreditation was determined if the unit was accredited by the Joint Commission for Accreditation of Healthcare Organizations.

Nursing Unit Environment-The predictor variable of nursing unit environment was defined by seven measures taken from the agency questionnaire. The number of RNs per patient within each perinatal hospice determined $R N$ unit size. Patient acuity was measure and derived from whether nurses cared for patients receiving continuous home care. $R N$ leadership was defined as a hospice director who held a nursing degree. $R N$ support services were defined by the presence of advanced practice nurses on staff at the perinatal hospice unit.

Nursing Unit Structure-The agency questionnaire revealed six categories which were used to define nursing unit structure. Calculation of the $R N$ proportion variable was performed by taking the number of full time RN employees (FTE) and dividing by the total number of RN and licensed practical nursing FTEs. $R N$ education was defined as the highest degree of employed RNs being a baccalaureate degree. Medical specialty certifications obtained determined the measurement of $R N$ expertise. Influenza vaccines being encouraged among the hospice nurses defined the safety climate. Career climate was obtained by a binary measure that determined clinical ladder availability. Finally, the technology climate was defined as a hospice nursing unit with available electronic medical records.

\section{Statistical Analysis}

The primary question of interest was whether there was an association between the nurse work environment and the delivery of culturally sensitive perinatal hospice care. For all analyses, data were weighted to reflect the population of hospice and home health agencies and to ensure adjustment for sampling bias. Descriptive statistics were obtained on the characteristics of culturally sensitive care and the nurse work environment. Due to the binary nature of the outcome variables, a multivariate logistic (logit) regression model was used to estimate the relationship between the delivery of culturally sensitive care and the nurse work environment. Individual regression analyses were conducted for each of the three indicators 
of culturally sensitive care. The logit regression model results are reported as adjusted odds ratios (ORs) with 95\% confidence intervals (CIs). Odds ratios are the chance of an outcome based on the presence of an independent variable. All analyses were conducted using Stata 11.0 software (Statcorp LP, College Station Texas).

\section{Results}

\section{Descriptive Statistics for Perinatal Hospices}

The characteristics of the nationally weighted number of perinatal hospices are summarized in Table 1. A majority of hospices provided interpreter services (87.3\%) and translation of materials $(68.1 \%)$. Less than half $(47.3 \%)$ had multilingual staff. Most hospices were nonfreestanding $(60.4 \%)$, located in a metropolitan area $(45.7 \%)$, and were small size $(88.9 \%)$. The average organizational age of the perinatal hospices was 16 years. It was common for hospices to be non-profit or government owned (84.9\%) and a clinical training site for students (87.5\%). Only $37.4 \%$ were accredited by the Joint Commission.

In the nursing unit environment, the average perinatal hospice had less than one $(\mu=0.66)$ full-time equivalent nurse per patient. Only $12.5 \%$ of agencies had patients who received continuous home care. It was common in perinatal hospices for the agency director to have a nursing degree (71.3\%). Less than a quarter of hospices (21.6\%) had nursing support staff in the form of clinical nurse specialists or nurse practitioners.

The nursing unit structure had on average a high proportion of RNs on staff $(\mu=0.87)$. Nurses were generally BSN educated (90.1\%) and had specialty certification (70\%). Perinatal hospice nurses typically worked on a safe (95.6\%) unit with technology (63.7\%), but only a quarter of hospices (24.9\%) offered nurses a positive career environment.

\section{Association Between Nurse Work Environment and Culturally Sensitive Care}

The analysis estimating the associations between the nurse work environment and the delivery of culturally sensitive care among perinatal hospices are shown in Table 2. With respect to interpreter services, the results revealed significant hospice environment relationships. The odds of offering interpreter services was almost four times higher for perinatal hospices that were a teaching site $(\mathrm{OR}=3.91,95 \% \mathrm{CI}$ : $1.48-10.29)$ compared to non-teaching hospices. Relative to non-accredited hospices, hospices accredited by the Joint Commission were positively associated with offering interpreter services to perinatal patients $(\mathrm{OR}=3.11,95 \% \mathrm{CI}$ : $1.00-9.72)$. No nursing unit environment or nursing unit structure characteristics were significantly associated with interpreter services.

The associations between the nurse work environment and translation of patient-related materials into other languages suggested the hospice environment and nursing unit structure were significantly related. There were higher odds $(\mathrm{OR}=1.06,95 \% \mathrm{CI}: 1.01-1.11)$ of translated materials in older perinatal agencies. Compared to non-teaching agencies, teaching perinatal hospices were 4 times more likely to have translated materials $(\mathrm{OR}=$ 3.93, 95\% CI: $1.40-11.01$ ). The odds of materials translated were more than double (OR = 2.46, 95\% CI: 1.20-5.06) among perinatal hospices with a technology climate of electronic 
medical records compared to those without. The nursing unit environment had no significant relationship with material translation.

With respect to multilingual perinatal staff, several nursing unit environment and structure characteristics were associated. Perinatal hospices with nursing-degreed agency directors were negatively associated with the presence of a multilingual staff. $\mathrm{RN}$ leadership (OR = $0.39,95 \%$ CI: $0.17-0.90$ ) was significantly and negatively associated with multilingual staff. For agencies with BSN-prepared nursing staff, the odds ratio (OR $=3.18$; 95\% CI: $1.16-$ 8.70) was significantly and positively related to having a multilingual staff compared to agencies without BSN-prepared nurses. The odds of having a multilingual staff were over twice ( $\mathrm{OR}=2.47,95 \% \mathrm{CI}: 1.15-5.33)$ for perinatal agencies with a technology climate compared to those without a technology climate. Multilingual staff among perinatal hospices was not significantly related to hospice environment characteristics.

\section{Discussion}

Our study evaluated the relationship between the nurse work environment and the delivery of culturally sensitive perinatal hospice care. Findings revealed that accreditation, teaching status, and baccalaureate prepared registered nurse staff influenced the provision of culturally sensitive care, regardless of hospice size.

Our combined finding was that an environment that embraces and stimulates education was more likely to offer culturally sensitive care for perinatal families. Valuing education and continued learning was demonstrated in the teaching status of a hospice organization and educated, baccalaureate-prepared registered nurse staff. One possible explanation is a learning environment inspires openness to differences and staff recognition that since culture is dynamic, one must engage in life-long learning (Institute of Medicine, 2010; Mixer, 2015). In addition, higher education contributes to comfort with cognitive dissonance and the perspective that there are multiple ways of providing care (Meyer and Xu, 2005). Taken together, these metacognitive factors, held at both the individual and organizational levels, may increase the likelihood of recognizing and responding to families' culture-specific needs. Future research might explore interventions on how best to train staff to provide culturally sensitive care and how to promote the value of continued learning at the nursing staff and organizational level.

Findings revealed that when perinatal hospices create a technology climate through electronic medical records, they are more likely to have translated patient-related materials and multilingual staff. Perinatal hospices with a technology climate may have the resources to support translated patient materials in languages of cultural groups in their service area (Kittler, 2004). Schmick et al. (2012) reported creating a customized Google ${ }^{\mathrm{TM}}$ Search Engine tool for health information that providers can query using English, which offers results in five different foreign languages used in local immigrant communities. To further cultural competence at the organizational level, future research should explore the importance of interpretive services in combination with materials translation. 


\section{Limitations}

The study has several limitations. Data were self-reported by hospices. Despite CDC quality checks, it is possible hospice administrators were reluctant to report negative data. Since the data were cross-sectional rather than longitudinal, no causal conclusions can be drawn. The depth and breadth of the 2007 Hospice and Homecare Survey data was a strength of this study because it provided the ability to examine detailed perinatal hospice characteristics. Conversely, given the rapidly changing landscape of culture and perinatal hospice care, the age of the data may also be viewed as a limitation. Unfortunately, 2007 was the last time this rich information was collected by the CDC. Investigating culture is complex, requiring multivariate longitudinal data. Therefore, without such comprehensive data collected, nurses and administrators may be challenged to measure the provision of culturally sensitive care.

\section{Implications}

Providing culturally sensitive hospice/palliative care for children and families is imperative (Weiner et al., 2013). Since administrators are responsible to provide the resources and staff dedicated to the provision of culturally sensitive care, the findings of our study have implications for nursing administration. The NCP Clinical Practice Guidelines for Quality Palliative Care and National CLAS Standards insist healthcare providers and organizations implement culturally and linguistically appropriate services (National Consensus Panel Clinical Practice Guidelines, 2013; United States Department of Health \& Human Services, 2014). Administrators and nursing staff may wish to investigate customized translated patient materials specific for local cultural groups served. While interpreter services, materials translated, and multilingual staff are necessary for culturally sensitive care, nurses must go beyond linguistic accommodations and demonstrate respect for and address the health values, beliefs, and practices of diverse families. Such a holistic, respectful approach can reduce health inequities and improve healthcare outcomes (United States Department of Health \& Human Services 2014). Additionally, administrators are encouraged to hire diverse applicants with strong qualifications who may serve as cultural brokers to families served in the region (Premji and Etowa, 2014).

In this study, $90 \%$ of perinatal hospice nursing staff were BSN educated nurses compared to reports that only 55-61\% of nurses in the U.S. hold a baccalaureate or higher degree (American Association of Colleges of Nursing, 2014a). Additionally, the AACN reports that nurses holding a BSN are "well prepared to meet the demands placed on today's nurse" and are "recognize[d for their] unique value ... [in] the practice setting" (American Association of Colleges of Nursing, 2014b). For hospices that have limited numbers of BSN educated staff, nurse administrators should consider incentives for formal education and training in delivery of culturally sensitive care.

Finally, the first step towards achieving culturally sensitive perinatal hospice care is assessing the unique needs of families. Cultural assessment provides a strong foundation upon which to build the therapeutic, trusting relationships between nurse and family that are necessary to navigate perinatal loss (Lauderdale, 2012; Weiner et al., 2013). Administrators and nursing staff can ensure cultural assessment is integrated into care and communicated to interdisciplinary colleagues. 


\section{Conclusion}

In conclusion, hospices in our study generally provided culturally and linguistically competent perinatal end-of-life care. This study suggests that the hospice and nursing unit environments, specifically in regards to education and technology, may be important contributors to the delivery of culturally sensitive care. Our study fills a gap in the literature, has implications for nursing staff and administrators, and suggests future research to support families experiencing perinatal loss.

\section{Acknowledgments}

Funding: This publication was made possible by Grant Number K01NR014490 from the National Institute of Nursing Research. Its contents are solely the responsibility of the authors and do not necessarily represent the official views of the National Institute of Nursing Research or National Institutes of Health.

\section{References}

American Association of Colleges of Nursing (AACN). Creating a more highly qualified nursing workforce. Fact Sheet. 2014a. http://www.aacn.nche.edu/media-relations/NursingWorkforce.pdf. (Accessed 18 October 2014)

American Association of Colleges of Nursing (AACN). The impact of education on nursing practice. Fact Sheet. 2014b. http://www.aacn.nche.edu/media-relations/fact-sheets/impact-of-education. (Accessed 18 October 2014)

Arthur KC, Mangione-Smith R, Meischke H, Zhou C, Strelitz B, Acosta Garcia M, Brown JC. Impact of English Proficiency on Care Experiences in a Pediatric Emergency Department. Academy Pediatrics. 2014; 15:218-24.

Balaguer A, Martín-Ancel A, Ortigoza-Escobar D, Escribano J, Argemi J. The model of palliative care in the perinatal setting: a review of the literature. BMC Pediatrics. 2012; 12:25. [PubMed: 22409881]

Barfield WD. Standard terminology for fetal, infant, and perinatal deaths. Pediatrics. 2011; 128:177181. [PubMed: 21708807]

Centers for Disease Control and Prevention. Survey methodology, documentation, and data files. 2011. http://www.cdc.gov/nchs/nhhcs/nhhcs_questionnaires.htm

Centers for Disease Control and Prevention. Guidance for Preventing Birth Defects. 2013a. http:// www.cdc.gov/ncbddd/birthdefects/data.html. (Accessed 7 July 2014)

Centers for Disease Control and Prevention. Preterm Birth. 2013b. http://www.cdc.gov/ reproductivehealth/maternalinfanthealth/pretermbirth.htm. (Accessed 10 July 2014)

Field, MJ.; Behrman, RE. Ethical conduct of clinical research involving children. Washington DC: The National Academies Press; 2011. http://www.nap.edu/openbook.php?record_id=10958. (Accessed 10 July 2014)

Goldenberg RL, Culhane JF, Iams JD, Romero R. Epidemiology and causes of preterm birth. Lancet. 2008; 371(9606):75-84. [PubMed: 18177778]

Hickerton CL, Aitken M, Hodgson J, Delatycki MB. "Did you find that out in time?": New life trajectories of parents who choose to continue a pregnancy where a genetic disorder is diagnosed or likely. Am J Med Genet Part A. 2012; 158:373-383. [PubMed: 22140089]

Institute of Medicine. The future of nursing: Leading change, advancing health. Robert Wood Johnson Foundation Initiative on the Future of Nursing at the Institute of Medicine. 2010. http:// www.iom.edu/reports/2010/the-future-of-nursing-leading-change-advancing-health.aspx

Irving C, Richmond S, Wren C, Longster C, Embleton ND. Changes in fetal prevalence and outcome for trisomies 13 and 18: A population-based study over 23 years. Journal of Maternal-Fetal and Neonatal Medicine. 2011; 24:137-141. [PubMed: 20384468] 
Kittler AF, Hobbs J, Volk LA, Kreps GL, Bates DW. The Internet as a vehicle to communicate health information during a public health emergency: A survey analysis involving the anthrax scare of 2001. Journal of Medical Internet Research. 2004; 6:e8-e12. [PubMed: 15111274]

Koh HK, Gracia JN, Alvarez ME. Culturally and Linguistically Appropriate Services - Advancing Health with CLAS. New England Journal of Medicine. 2014; 371:198-201. [PubMed: 25014685]

Lauderdale, J. Transcultural perspectives in childbearing. In: Andrews, MM.; Boyle, JS., editors. Transcultural Concepts in Nursing Care. 6th. Philadelphia: Wolters Kluwer Health/Lippincott Williams \& Wilkins; 2012. p. 91-119.

Lindley LC, Fornehed ML, Mixer SJ. A comparison of the nurse work environment between perinatal and non-perinatal hospice providers. International journal of palliative nursing. 2013; 19:535-540. [PubMed: 24263897]

Lion KC, Rafton SA, Shafii J, Brownstein D, Michel E, Tolman M, Ebel BE. Association between language, serious adverse events, and length of stay among hospitalized children. Hospital Pediatrics. 2013; 3:219-225. [PubMed: 24313090]

Macdorman M, Kirmeyer S, Wilson E. Fetal and perinatal mortality, United States, 2006. Hyattsville, MD, National Center for Health Statistics. National vital statistics reports. 2012; 60(8):23.

McFarland, MR.; Wehbe-Alamah, H. The theory of culture care diversity and universality. In: McFarland, MR.; Wehbe-Alamah, HB., editors. Leininger's Culture Care Diversity and Universality: A Worldwide Nursing Theory. 3rd. New York: Jones \& Bartlett; 2015. p. 1-34.

Meyer T, Xu Y. Academic and clinical dissonance in nursing education: Are we guilty of failure to rescue? Nurse Educator. 2005; 30:76-79. [PubMed: 15785345]

Mixer, SJ. Application of culture care theory in teaching cultural competence and culturally congruent care. In: McFarland, MR.; Wehbe-Alamah, HB., editors. Leininger's Culture Care Diversity and Universality: A Worldwide Nursing Theory. 3rd. New York: Jones \& Bartlett; 2015. p. 369-385.

National Consensus Panel Clinical Practice Guidelines. Clinical practice guidelines for quality palliative care. 2013. http://www.nationalconsensusproject.org/Guidelines_Download2.aspx. (Accessed 1 August 2014)

Premji S, Etowa JB. Workforce utilization of visible and linguistic minorities in Canadian nursing. Journal of Nursing Management. 2014; 22:80-88. [PubMed: 23410219]

Schmick DD, Johnson ED, Scoville CL, Vaduvathiriyan PK. Building a Google custom search engine (CSE) for foreign language health information: One library's effort to create a new tool for health professionals. Journal of Consumer Health on the Internet. 2012; 16:27-36.

Sumner LH, Kavanaugh K, Moro T. Extending palliative care into pregnancy and the immediate newborn period: state of the practice of perinatal palliative care. The Journal of Perinatal \& Neonatal Nursing. 2006; 20:113-116. [PubMed: 16508478]

United States Department of Health and Human Services. National Home and Hospice Care Survey, 2007. Ann Arbor, MI: Inter-university Consortium for Political and Social Research (distributor); 2010. Centers for Disease Control and Prevention. National Center for health Statistics. ICPSR28961-v12010-09-01. http://www.icpsr.umich.edu/icpsrweb/NACDA/studies/28961/ version/1

United States Department of Health \& Human Services Office of Minority Health. Think cultural health. 2014. https://www.thinkculturalhealth.hhs.gov/content/clas.asp. (Accessed 17 October 2014)

Weiner L, McConnell DG, Latella L, Ludi E. Cultural and religious considerations in pediatric palliative care. Palliative and Supportive Care. 2013; 11:47-67. [PubMed: 22617619]

Wool C. State of the science on perinatal palliative care. J Obstet Gynecol Neonatal Nurs. 2013; 42:372-382.

Wool C. Clinician perspectives on barriers to perinatal palliative care. MCN Am J Matern Child Nurs. 2015; 40:44-50. [PubMed: 25503834] 
Table 1

Descriptive Statistics of Study Variables Among Perinatal Hospices (Weighted N=995)

\begin{tabular}{|c|c|c|c|c|}
\hline Characteristics & Number & Mean/Proportion & Min & $\operatorname{Max}$ \\
\hline \multicolumn{5}{|c|}{ Culturally Sensitive Care } \\
\hline Interpreter Services & 869 & $87.3 \%$ & 0 & 1 \\
\hline Materials Translated & 678 & $68.1 \%$ & 0 & 1 \\
\hline Multilingual Staff & 471 & $47.3 \%$ & 0 & 1 \\
\hline \multicolumn{5}{|l|}{ Hospice Environment } \\
\hline \multicolumn{5}{|l|}{ Affiliation } \\
\hline Non-Freestanding & 601 & $60.4 \%$ & 0 & 1 \\
\hline Freestanding & 394 & $39.6 \%$ & 0 & 1 \\
\hline \multicolumn{5}{|l|}{ Service Area } \\
\hline Metropolitan & 455 & $45.7 \%$ & 0 & 1 \\
\hline Micropolitan & 305 & $30.6 \%$ & 0 & 1 \\
\hline Rural & 235 & $23.7 \%$ & 0 & 1 \\
\hline \multicolumn{5}{|l|}{ Facility Size } \\
\hline Small & 880 & $88.9 \%$ & 0 & 1 \\
\hline Large & 115 & $11.1 \%$ & 0 & 1 \\
\hline Organizational Age & 995 & 16.13 & 14.47 & 17.79 \\
\hline \multicolumn{5}{|l|}{ Ownership } \\
\hline Non-profit/Govt. & 845 & $84.9 \%$ & 0 & 1 \\
\hline For-profit & 150 & $15.1 \%$ & 0 & 1 \\
\hline Teaching Status & 870 & $87.5 \%$ & 0 & 1 \\
\hline Accreditation & 372 & $37.4 \%$ & 0 & 1 \\
\hline \multicolumn{5}{|c|}{ Nursing Unit Environment } \\
\hline RN Unit Size & 995 & 0.66 & 0.52 & 0.80 \\
\hline Patient Acuity & 124 & $12.5 \%$ & 0 & 1 \\
\hline RN Leadership & 709 & $71.3 \%$ & 0 & 1 \\
\hline RN Support Services & 215 & $21.6 \%$ & 0 & 1 \\
\hline \multicolumn{5}{|l|}{ Nursing Unit Structure } \\
\hline RN Proportion & 995 & 0.87 & 0.85 & 0.90 \\
\hline RN Education -BSN & 896 & $90.1 \%$ & 0 & 1 \\
\hline RN Expertise & 697 & $70.0 \%$ & 0 & 1 \\
\hline Safety Climate & 951 & $95.6 \%$ & 0 & 1 \\
\hline Career Climate & 248 & $24.9 \%$ & 0 & 1 \\
\hline Technology Climate & 634 & $63.7 \%$ & 0 & 1 \\
\hline
\end{tabular}

Int J Palliat Nurs. Author manuscript; available in PMC 2016 September 30. 
Table 2

Regression Analysis for Delivery of Culturally Sensitive Care Among Perinatal Hospices (Weighted N=995)

\begin{tabular}{|c|c|c|c|}
\hline & $\begin{array}{l}\text { Interpreter Services } \\
\text { OR }(95 \% \mathrm{CI})\end{array}$ & $\begin{array}{l}\text { Materials Translated } \\
\text { OR(95\% CI })\end{array}$ & $\begin{array}{l}\text { Multilingual Staff } \\
\text { OR(95\% CI) }\end{array}$ \\
\hline \multicolumn{4}{|l|}{ Hospice Environment } \\
\hline Freestanding & $2.02(0.72-5.71)$ & $1.12(0.52-2.39)$ & $1.85(0.88-3.87)$ \\
\hline \multicolumn{4}{|l|}{ Service Area } \\
\hline Metropolitan & (ref) & (ref) & (ref) \\
\hline Micropolitan & $1.20(0.35-4.14)$ & $1.01(0.41-2.51)$ & $1.35(0.56-3.27)$ \\
\hline Rural & $1.43(0.37-5.59)$ & $1.53(0.53-4.43)$ & $1.38(0.56-3.40)$ \\
\hline Large Size & $0.45(0.86-2.39)$ & $0.63(0.21-1.87)$ & $2.55(0.82-7.91)$ \\
\hline Organizational Age & $1.03(0.98-1.08)$ & $1.06(1.01-1.11)^{*}$ & $1.01(0.96-1.07)$ \\
\hline For-profit & $2.57(0.73-9.04)$ & $2.11(0.77-5.79)$ & $1.27(0.44-3.70)$ \\
\hline Teaching Status & $3.91(1.48-10.29)^{* *}$ & $3.93(1.40-11.01)^{* *}$ & $1.61(0.62-4.23)$ \\
\hline Accreditation & $3.11(1.00-9.72)^{*}$ & $0.98(0.47-2.08)$ & $1.75(0.79-3.88)$ \\
\hline \multicolumn{4}{|c|}{ Nursing Unit Environment } \\
\hline RN Unit Size & $1.40(0.60-3.25)$ & $0.75(0.54-1.05)$ & $0.83(0.58-1.18)$ \\
\hline Patient Acuity & $1.30(0.41-4.12)$ & $0.71(0.29-1.78)$ & $0.62(0.27-1.41)$ \\
\hline RN Leadership & $0.77(0.27-2.14)$ & $0.76(0.35-1.66)$ & $0.39(0.17-0.90)^{*}$ \\
\hline RN Support Services & $3.43(0.77-15.23)$ & $1.49(0.60-3.74)$ & $0.60(0.21-1.74)$ \\
\hline \multicolumn{4}{|c|}{ Nursing Unit Structure } \\
\hline RN Proportion & $4.55(0.18-116.78)$ & $2.40(0.26-22.52)$ & $0.28(0.03-2.46)$ \\
\hline RN Education -BSN & $1.37(0.51-3.66)$ & $2.19(0.89-5.28)$ & $3.18(1.16-8.70)^{*}$ \\
\hline Expertise & $1.89(0.67-5.38)$ & $1.13(0.47-2.70)$ & $0.89(0.36-2.19)$ \\
\hline Safety Climate & $2.15(0.31-15.04)$ & $1.13(0.23-5.59)$ & $0.44(0.09-2.26)$ \\
\hline Career Climate & $1.97(0.55-7.08)$ & $2.02(0.87-4.72)$ & $2.72(0.99-7.51)$ \\
\hline Technology Climate & $2.26(0.84-6.04)$ & $2.46(1.20-5.06)^{*}$ & $2.47(1.15-5.33)^{*}$ \\
\hline
\end{tabular}

Note. $\mathrm{OR}=$ odds ratio. $\mathrm{CI}=$ confidence interval. $\mathrm{RN}=$ registered nurse $. \mathrm{BSN}=$ Bachelors of Science in Nursing. * $p<0.05$,

*** $p<0.01$ 